\title{
Método simplificado versus convencional de confecção de próteses totais: práticas adotadas pelos Cirurgiões-Dentistas
}

\author{
Simplified versus conventional method for the fabrication of complete \\ dentures: procedures adopted by dentists
}

\author{
Thais Angelina CAETANO ${ }^{a}$, Adriana Barbosa RIBEIRO, Maria Paula DELLA VECCHIA ${ }^{a}$, \\ Tatiana Ramirez CUNHA a , Raphael Freitas de SOUZA ${ }^{a}$
}

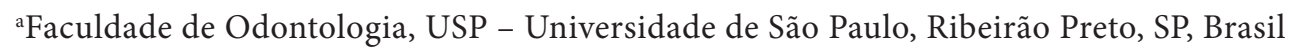

\begin{abstract}
Resumo
Introdução: A fim de reduzir custos e tempo clínico envolvidos com o tratamento, Dentistas têm utilizado métodos simplificados, omitindo ou abreviando partes da técnica de confecção das próteses totais. Objetivo: Levantar as práticas utilizadas pelos Cirurgiões-Dentistas para a confecção de próteses totais. Material e método: Os dados foram levantados por meio de um formulário eletrônico contendo perguntas relativas ao perfil e aos procedimentos clínicos utilizados, a fim de quantificar o uso de métodos simplificados e convencionais, bem como para determinar a relação com o perfil desses profissionais. Os resultados foram analisados por meio de análise de Aglomerados. Resultado: As respostas foram dadas, em sua maioria, por homens, tendo a prática clínica como sua principal fonte de renda e clínica privada ou conveniada como ambiente principal de trabalho $(\mathrm{n}=119)$. Grande parte dos entrevistados relatou realizar duas moldagens por arcada, utilização de articulador semiajustável com arco facial, determinação de plano oclusal por meio de guias anatômicos, utilização de meios métricos para análise da dimensão vertical de oclusão e determinação direta da relação cêntrica. O próprio Cirurgião-Dentista relatou ser o responsável na fase de seleção de dentes. A estatística identificou dois Aglomerados, com base nas características demográficas e na prática clínica, sendo um grupo formado por generalistas adeptos de perfis mais simplificados e outro formado por profissionais mais especializados em prótese que realizam técnicas convencionais. Conclusão: A adoção de técnicas simplificadas é associada àqueles Dentistas com perfil mais generalista, enquanto protesistas tendem a trabalhar mais de acordo com a Academia.
\end{abstract}

Descritores: Prótese total; coleta de dados; tecnologia biomédica.

\begin{abstract}
Introduction: In order to reduce costs and clinical time involved with complete denture treatment, dentists have employed simplified methods based on the reduction or preclusion of certain traditional technical procedures. Purpose: To survey the procedures used by dentists to fabricate complete dentures. Material and method: Data was obtained by an electronic form containing questions about dentists' profile and clinical procedures, in order to quantify traditional and simplified approaches at several moments and to determine them to different professional profiles. Results were evaluated by means of cluster analysis. Result: The majority of respondents were male dentists with clinical practice as their main income source, mainly in private service $(\mathrm{n}=119)$. Most participants use to take a pair of impressions from each arch, employ semiadjustable articulators with facebow transfers, shape the occlusal plane with the aid of anatomic references, determine occlusal vertical dimension by metric methods and use mandibular manipulation for centric relation. The dentists performed tooth selection in most cases. Statistical analysis identified two clusters based on demographic characteristics and clinical procedures. General practitioners adopting simplified methods formed the first cluster, whereas prosthodontists used to conventional methods formed the second. Conclusion: General dentists tend to adopt simplified approaches, and prosthodontists tend to work according to traditional academic methods.
\end{abstract}

Descriptors: Denture, complete; data collection; biomedical technology.

\section{INTRODUÇÃO}

O edentulismo completo pode ser considerado um problema importante de saúde pública, particularmente entre os idosos ${ }^{1}$, sendo que sua prevalência entre adultos com 65 ou mais anos de idade é alta em vários países, como Canadá (58\%), Arábia Saudita (31-46\%), Reino Unido (46\%), China (11\%) e Índia $(19 \%)^{2}$. Estima-se que $37,5 \%$ da população entre 65 e 74 anos de 
idade utilizam próteses totais nos arcos maxilar e mandibular, respectivamente, e que a necessidade de próteses totais duplas em brasileiros idosos é de $38,3 \%{ }^{3}$.

Os métodos convencionais para confecção de próteses totais exigem uma ampla sequência de procedimentos clínicos e laboratoriais ${ }^{4,5}$, e têm sido aceitos e ensinados pela maioria das faculdades de Odontologia no Brasil ${ }^{6}$ e nos Estados Unidos ${ }^{7}$. Por outro lado, a literatura apresenta questionamentos sobre a real necessidade de toda a sequência desses métodos para a obtenção de próteses totais adequadas. Algumas abordagens têm sido descritas no intuito de simplificar etapas clínicas e laboratoriais, como a confecção de bases de prova diretamente sobre modelos de estudo obtidos a partir de moldes de alginato feito com moldeiras de estoque, ou seja, sem uma segunda moldagem ${ }^{8}$. Outras abordagens simplificadas incluem a montagem dos modelos em articulador sem o uso do arco facial ${ }^{9}$, os esquemas oclusais alternativos como a oclusão lingualizada ${ }^{10} \mathrm{e}$ as técnicas baseadas na duplicação de próteses em uso ${ }^{11}$.

Alguns ensaios clínicos controlados randomizados recentes têm fornecido informações de alto nível de evidência referentes à eficácia de métodos simplificados. Em setembro de 2013, realizou-se uma busca sistemática na base PubMed, conforme descrito por Della Vecchia et al. ${ }^{12}$. Dentre os 531 títulos e resumos resultantes, foram encontrados alguns relatos de ensaios clínicos randomizados que tentaram avaliar métodos simplificados para a confecção de próteses totais e compará-los aos métodos tradicionais $^{12-18}$. Três desses ensaios buscaram comparar a ausência de uma segunda moldagem (funcional com moldeira de estoque) e do uso do arco facial, no Brasil ${ }^{12,13,18}$, Canadá ${ }^{15,16} \mathrm{e}$ Kuwait $^{17}$. Outro ensaio comparou próteses totais confeccionadas de acordo com dois conceitos oclusais ${ }^{14}$. Em todos os casos, os métodos convencionais não resultaram em diferentes resultados em termos de satisfação do paciente e qualidade das próteses. Porém, o custo e o tempo envolvidos na confecção de próteses totais pelo método convencional foram significativamente maiores em relação ao simplificado, indicando que o método simplificado é de melhor relação custo-efetividade $e^{12,13,15-18}$.

Mesmo antes de possíveis vantagens terem sido estudadas, o uso do método simplificado já havia sido difundido entre muitos clínicos nos Estados Unidos e no Reino Unido. Nesses países, boa parte dos Cirurgiões-Dentistas não utiliza rotineiramente o método convencional para a confecção de próteses totais e sim algum método simplificado ${ }^{19,20}$. Estes métodos simplificados são eleitos por esses profissionais porque são mais fáceis de dominar do que a técnica tradicional, além de reduzirem custos e tempo envolvidos na confecção das próteses totais ${ }^{16}$.

Assim, uma pergunta pode ser feita: um método simplificado parece aceitável à classe odontológica, em nosso país? Apesar dos estudos citados, uma resistência por adotar técnicas simplificadas por parte dos profissionais poderia ser esperada, dada a grande difusão da sequência convencional nas universidades ${ }^{6,7}$. Ainda, o tempo longo de experiência clínica com a técnica convencional poderia contribuir com uma postura mais conservadora em relação à aquisição de novos métodos.
Este estudo tem por objetivo o levantamento de práticas utilizadas pelos Cirurgiões-Dentistas para a confecção de próteses totais. Dados foram levantados por meio de formulário eletrônico (via internet), a fim de quantificar o uso de métodos simplificados e convencionais, bem como para determinar o perfil dos profissionais.

\section{MATERIAL E MÉTODO}

Este trabalho envolve um levantamento de informações a partir de Cirurgiões-Dentistas brasileiros. Formulários foram enviados via e-mail, contendo perguntas relativas ao perfil profissional e aos procedimentos clínicos usados para a obtenção de próteses totais convencionais. O presente estudo obedeceu a um desenho observacional do tipo transversal, em que apenas uma coleta de dados foi feita por profissional consultado. Por fim, a análise estatística investigou uma associação entre os procedimentos clínicos e o perfil dos Dentistas avaliados.

\section{Coleta de Dados}

Os dados foram coletados por meio de um formulário eletrônico no período de fevereiro a maio de 2012. Esse formulário ficou armazenado na plataforma Google e foi acessado pelos participantes por meio de uma ligação fornecida via e-mail. O e-mail continha os objetivos do estudo e o número de perguntas a serem administradas. Também explicitava a natureza sigilosa dos dados coletados. Ressalte-se que este trabalho foi iniciado somente após apreciação pelo Comitê de Ética em Pesquisa institucional (CAAE: 0019.0.138.000-11).

Assim que o possível participante clicasse na ligação, a página da internet era aberta, mostrando 18 perguntas, sendo 14 destas com respostas fechadas e quatro com respostas abertas (Quadro 1). Em sumário, as questões foram direcionadas a aspectos demográficos e aos seguintes domínios: (1) Técnica de moldagem utilizada; (2) Uso do articulador e arco facial; (3) Determinação dos planos de orientação; (4) Dimensão vertical de oclusão; (5) Relação central; (6) Provas clínicas das placas articulares com dentes montados; (7) Remontagem das próteses totais finalizadas.

Uma versão preliminar desse questionário foi testada em dez Cirurgiões-Dentistas, dentre eles graduados, pós-graduados e doutores em Odontologia. Durante essa etapa pré-teste, foi verificado se as questões eram compreensíveis, se estavam ordenadas de maneira lógica e se o tempo para preenchimento era adequado. A Tabela 1 resume as características dos colaboradores dessa etapa.

Após o pré-teste, o questionário foi enviado aos potenciais participantes. $\mathrm{O}$ envio dos questionários foi realizado por meio de uma empresa de publicidade específica do ramo odontológico e que continha um cadastro de Cirurgiões-Dentistas. As respostas coletadas foram armazenadas em servidor, para posterior organização em planilhas no programa Microsoft Excel 2003 (Microsoft Corporation, São Paulo, SP). 
Quadro 1. Questionário enviado aos participantes e respostas possíveis, quando aplicável

1. Gênero

(a) Masculino; (b) Feminino

2. Quantas horas semanais você trabalha na clínica, independentemente do tipo de atendimento realizado?

3. Quantos pacientes você atende por dia, aproximadamente?

4. Com que frequência você realiza tratamento com próteses totais convencionais?

(a) Quase todos os dias; (b) Frequentemente; (c) Às vezes; (d) Raramente; (e) Nunca

5. Sua prática clínica é a principal fonte de renda, sua ou de sua família?

(a) Sim; (b) Não

6. Sua principal atividade clínica acontece em que ambiente?

(a) Clínica privada ou conveniada; (b) Saúde pública; (c) Ambas

7. Qual a seqüência clínica que o(a) Sr(a) adota para a moldagem dos pacientes que receberão próteses totais?

(a) Moldagem única por arcada para a obtenção de modelos de trabalho, com materiais como alginato ou godiva em placa; (b) Duas moldagens por arcada (ex.: uma preliminar e outra funcional) para a obtenção de modelos de estudo/anatômicos e posteriormente modelos de trabalho; (c) Uso de próteses antigas como moldeira na obtenção de modelo de trabalho; (d) Outras; (e) Não sei responder

8. Considerando o tipo de articulador usado para a montagem de modelos de trabalho, qual alternativa descreve melhor sua prática clínica?

(a) Uso de charneira ou outro articulador não ajustável; (b) Montagem em articulador semiajustável sem arco facial; (c) Montagem em articulador semiajustável com uso do arco facial; (d) Não utilizo articulador; (e) Não sei responder.

9. Como o(a) Senhor(a) determina os planos de orientação/plano oclusal das futuras próteses totais?

(a) Por meio de guias anatômicos (ex.: como a linha bipupilar, plano de Camper, plano de Frankfurt, plano entre papilas retromolares e comissuras labiais, ou outros); (b) Por meio de critérios visuais/experiência clínica; (c) Não sei responder

10. Como o(a) Senhor(a) determina a dimensão vertical de oclusão?

(a) Usando métodos métricos (ex.: proporção dos terços faciais) e/ou fisiológicos (ex.: método fonético, deglutição), associados ou não à observação arbitrária; (b) Somente pela visualização da aparência facial e/ou checando o conforto do paciente; (c) Não sei responder.

11. Como o(a) Senhor(a) determina a relação cêntrica/posição de trabalho no sentido horizontal?

(a) Usando algum dispositivo (ex.: pelo registro do arco gótico); (b) Determinação direta sobre os roletes de cera/placas articulares (ex.: método guiado ou determinação de uma posição confortável); (c) Não sei responder.

12. Quem realiza a seleção dos dentes artificiais na maioria dos casos clínicos?

(a) O(a) Senhor (a) Cirurgião(ã)-Dentista; (b) Técnico de prótese dentária/laboratório; (c) Não sei responder.

13. Como realiza a sessão de provas clínicas das bases com dentes montados (antes da acrilização)?

(a) Nenhuma prova (o técnico monta os dentes e acriliza as próteses sem provas anteriores); (b) Uma prova somente (após montagem de todos ou parte dos dentes, é feita uma sessão de prova e em seguida a acrilização); (c) Duas provas (realiza uma prova após montagem de alguns dentes, com os dentes anteriores, e uma segunda com os posteriores); (d) Três ou mais provas clínicas; (e) Não sei responder.

14. O(a) Senhor(a) realiza remontagem das próteses totais acrilizadas para ajuste da oclusão?

(a) Sim, as próteses totais são remontadas e ajustadas em articulador; (b) Não. Ajusto a oclusão exclusivamente na boca do paciente; (c) Não sei responder.

15. Quantas sessões clínicas o(a) Senhor(a) acha que seria ideal para a confecção de próteses totais convencionais, contando desde a moldagem inicial até a sessão de instalação?

16. O(a) Senhor(a) acha que o número de sessões clínicas envolvidas no tratamento com próteses totais é um fator importante do ponto de vista do paciente?

(a) Sim; (b) Não; (c) Não sei responder

17. Ano em que concluiu o Curso de Graduação.

18. Grau mais alto de formação.

(a) Graduação em Odontologia; (b) Especialização em Prótese Dentária; (c) Especialização em outra área; (d) Mestrado e/ou Doutorado em prótese dentária; (e) Mestrado e/ou Doutorado em outra área 
Tabela 1. Características dos Dentistas colaboradores na fase préteste

\begin{tabular}{|ccc|}
\hline Colaborador & Ano de graduação & $\begin{array}{c}\text { Especialista em } \\
\text { Prótese Dentária }\end{array}$ \\
\hline 1 & 2010 & Não \\
\hline 2 & 2010 & Não \\
\hline 3 & 1992 & Sim \\
\hline 4 & 1996 & Sim \\
\hline 5 & 1973 & Sim \\
\hline 6 & 1984 & Sim \\
\hline 7 & 2005 & Não \\
\hline 8 & 2005 & Sim \\
\hline 9 & 1983 & Não \\
\hline 10 & 1987 & Sim \\
\hline
\end{tabular}

Dentro do formulário de avaliação, constam questões que levaram à exclusão de possíveis participantes. Nesse caso, foram excluídos Dentistas que: (1) Não exerciam a clínica odontológica regularmente; (2) Não confeccionavam próteses totais em sua prática clínica; (3) Recusaram-se a preencher o formulário após leitura do parágrafo introdutório do e-mail, análogo a um termo de consentimento livre e esclarecido.

\section{Análise dos Dados}

Foi realizada uma análise descritiva dos dados coletados, resumindo-os por meio de contagens de frequência em tabelas e gráficos. Uma determinação do perfil demográfico em função do uso de técnicas simplificadas ou convencionais foi feita por meio de análise de Aglomerados (cluster analysis). Para esse teste, empregaram-se procedimentos similares àqueles descritos por Nunes et al. ${ }^{21}$, e buscou-se uma amostra de 100 participantes antes da análise. As análises foram realizadas com um nível de significância de 0,05 por meio do software PASW 18.0 (SPSS Inc., Chicago, IL, EUA).

\section{RESULTADO}

Dentre os profissionais cadastrados em banco de dados (dado não fornecido), 122 Cirurgiões-Dentistas responderam ao questionário on line. A Tabela 2 refere-se às suas características demográficas. A maioria dos participantes era do gênero masculino (62\%), tinha a prática clínica odontológica como principal fonte de renda (82\%), clínica privada ou conveniada como principal ambiente de trabalho (89\%) e o tempo médio desde a graduação era 18,8 anos. A jornada semanal em clínica era, em média, 35 horas e a média diária de pacientes atendidos era dez. A maioria dos respondentes trabalhava com próteses totais frequentemente (39\%) e ocasionalmente (32\%). Os participantes sugeriram que o número ideal de sessões necessárias para confecção de uma prótese total seria de aproximadamente cinco
Tabela 2. Características demográficas da amostra do estudo

\begin{tabular}{lc}
\hline \multicolumn{1}{|c}{ Características } & Total \\
\hline Gênero & 75 \\
\hline - Masculino & 47 \\
\hline - Feminino & $34,7 \pm 15,7$ \\
\hline Horas semanais trabalhadas na clínica odontologica $\dagger$ & $9,9 \pm 7,1$ \\
\hline Pacientes atendidos por dia $\dagger$ & \\
\hline Frequencia na realização de próteses totais & 5 \\
\hline - Nunca & 24 \\
\hline - Raramente & 39 \\
\hline - Às vezes & 48 \\
\hline - Frequentemente & 6 \\
\hline - Quase todos os dias & \\
\hline Prática clínica como principal renda & $18,8 \pm 10,6$ \\
\hline - Sim & 100 \\
\hline - Não & 22 \\
\hline Ambiente de atividade clínica & $6,3 \pm 1,2$ \\
\hline - Clínica privada ou conveniada & 7 \\
\hline - Saúde pública & \\
\hline - Ambas & \\
\hline Número de sessões ideais $\dagger$ & \\
\hline Tempo de formação (anos) $\dagger$ & \\
\hline$\dagger$ Valores expressos em média \pm desvio padrão. & \\
\hline
\end{tabular}

sessões, em média. Dentre os participantes, três foram excluídos da análise estatística, pois não realizavam a confecção de próteses totais como uma prática clínica odontológica, apesar de terem fornecido dados demográficos. Sendo assim, as respostas de 119 Cirurgiões-Dentistas foram consideradas para análise de Aglomerados.

A maioria das respostas sobre a sequência de moldagem recomendou duas sessões. Quanto ao uso de articuladores, boa parte da amostra relatou uso de modelos semiajustáveis conjugados ao uso do arco facial (36\%). A determinação de planos de orientação/oclusal por meio de guias anatômicos (79\%) e meios métricos para determinação da dimensão vertical de oclusão (81\%) foram relatados com frequência; o maior número de respostas sobre a relação cêntrica se concentrou na forma de determinação direta ( $88 \%$ ), ou seja, sem a utilização de dispositivos. Relacionado à seleção de dentes, o próprio Cirurgião-Dentista assumiu tal responsabilidade em 62\%. Uma sessão para prova clínica dos dentes montados e ausência de remontagem das próteses acrilizadas para ajuste de oclusão foram maioria nas respostas obtidas. Ainda, 84\% dos respondentes apontaram o número de sessões clínicas como fator importante na confecção de uma prótese total.

Asfrequências derespostas eos resultados da análise inferencial podem ser observados na Tabela 3 , sendo que os participantes 
Tabela 3. Distribuição dos Dentistas conforme as características demográficas e a prática clínica

\begin{tabular}{|c|c|c|c|}
\hline \multirow{2}{*}{ Variável } & \multirow{2}{*}{ Categorias } & \multicolumn{2}{|c|}{ Aglomerados } \\
\hline & & 1 & 2 \\
\hline Horas semanais em clínica & - & $35,8 \pm 15,7$ & $33,7 \pm 15,7$ \\
\hline Pacientes por dia & - & $11,8 \pm 9,0$ & $8,3 \pm 4,3$ \\
\hline Número de sessões ideais & - & $4,9 \pm 0,9$ & $5,9 \pm 1,2$ \\
\hline Anos de formação & - & $17,2 \pm 8,7$ & $20,1 \pm 11,9$ \\
\hline \multirow{2}{*}{ Gênero } & Feminino & 27 & 19 \\
\hline & Masculino & 29 & 44 \\
\hline \multirow{5}{*}{$\begin{array}{l}\text { Frequência de tratamento com } \\
\text { próteses totais }\end{array}$} & Nunca & 2 & 0 \\
\hline & Raramente & 10 & 14 \\
\hline & Às vezes & 21 & 18 \\
\hline & Frequentemente & 21 & 27 \\
\hline & Quase todos os dias & 2 & 4 \\
\hline \multirow{2}{*}{ Clínica é principal fonte de renda? } & Sim & 16 & 51 \\
\hline & Não & 10 & 12 \\
\hline \multirow{3}{*}{ Ambiente clínico } & Particular ou convênio & 18 & 59 \\
\hline & Saúde pública & 3 & 4 \\
\hline & Ambas & 5 & 0 \\
\hline \multirow{5}{*}{ Moldagem } & Uma sessão & 15 & 4 \\
\hline & Duas sessões & 40 & 56 \\
\hline & Sobre PT antiga & 0 & 1 \\
\hline & Outras & 0 & 2 \\
\hline & Não sabe & 1 & 0 \\
\hline \multirow{5}{*}{$\begin{array}{l}\text { Montagem dos modelos de } \\
\text { trabalho }\end{array}$} & Não usa & 11 & 1 \\
\hline & Charneira & 28 & 5 \\
\hline & ASA sem arco & 11 & 18 \\
\hline & ASA com arco & 4 & 39 \\
\hline & Não sabe & 2 & 0 \\
\hline \multirow{3}{*}{ Determinação do plano oclusal } & Visualmente & 20 & 3 \\
\hline & Guias anatômicos & 35 & 60 \\
\hline & Não sabe & 1 & 0 \\
\hline \multirow{3}{*}{ Dimensão Vertical } & Visualmente & 20 & 0 \\
\hline & Métricos & 35 & 63 \\
\hline & Não sabe & 1 & 0 \\
\hline \multirow{3}{*}{ Relação Cêntrica } & Determinação direta & 54 & 52 \\
\hline & Dispositivo & 0 & 11 \\
\hline & Não sabe & 2 & 0 \\
\hline \multirow{3}{*}{ Seleção dos dentes } & Técnico & 40 & 4 \\
\hline & Dentista & 15 & 49 \\
\hline & Não sabe & 1 & 0 \\
\hline
\end{tabular}


Tabela 3. Continuação...

\begin{tabular}{|c|c|c|c|}
\hline \multirow{2}{*}{ Variável } & \multirow{2}{*}{ Categorias } & \multicolumn{2}{|c|}{ Aglomerados } \\
\hline & & 1 & 2 \\
\hline \multirow{4}{*}{ Sessões de provas clínicas } & Uma & 45 & 44 \\
\hline & Duas & 5 & 8 \\
\hline & Três ou mais & 4 & 11 \\
\hline & Não sabe & 2 & 0 \\
\hline \multirow{3}{*}{ Remontagem } & Não & 49 & 39 \\
\hline & Sim & 4 & 23 \\
\hline & Não sabe & 3 & 1 \\
\hline \multirow{3}{*}{ Importância do número de sessões } & Não & 12 & 5 \\
\hline & Sim & 43 & 58 \\
\hline & Não sabe & 1 & 0 \\
\hline \multirow{5}{*}{ Grau de formação } & Graduação & 11 & 9 \\
\hline & Especialização em outra área & 29 & 17 \\
\hline & Especialização em Prótese & 6 & 13 \\
\hline & Stricto sensu em outra área & 9 & 10 \\
\hline & Stricto sensu em Prótese & 1 & 14 \\
\hline
\end{tabular}

foram divididos em dois Aglomerados definidos, conforme a semelhança nas respostas. No Aglomerado 1, pode-se observar uma heterogeneidade quanto ao gênero; já no Aglomerado 2, há uma concentração maior no gênero masculino, maior tempo de formação dos participantes e predominância da clínica particular ou conveniada como principal ambiente de trabalho. Referente às horas semanais trabalhadas em clínica, os dois grupos tiveram uma diferença muito pequena nas respostas, obtendo-se médias de 36 e 34 horas, respectivamente. O Aglomerado 1 parece atender uma maior quantidade de pacientes ao dia em relação ao Aglomerado 2; quanto à frequência no tratamento com próteses totais, os dois grupos apresentaram respostas semelhantes, variando nas respostas: 'raramente, às vezes' e 'frequentemente.' $\mathrm{O}$ Aglomerado 2 também relatou a proposta da necessidade de um maior número de sessões ideais na confecção de próteses totais, quando comparado ao Aglomerado 1; ainda no Aglomerado 2, um maior número de pessoas respondeu 'sim' em relação à clínica como sua principal fonte de renda.

Quanto à prática clínica, nos dois Aglomerados, o maior número de respostas foi dado pela realização de duas sessões de moldagem; no entanto, alguns indivíduos do grupo 1 relataram a realização de apenas uma sessão. Na montagem dos modelos de trabalho, o Aglomerado 1 relatou usar mais articuladores do tipo charneira, enquanto o Aglomerado 2 usa predominantemente o articulador semiajustável (ASA) com arco facial. Em relação à determinação do plano oclusal e da dimensão vertical de oclusão, no Aglomerado 1, houve heterogeneidade nas respostas; já no Aglomerado 2, predominou o uso de guias anatômicos e métricos. Uma maior concentração de respostas quanto à determinação direta da relação cêntrica está no Aglomerado 1, enquanto que, no Aglomerado 2, pequena parte relatou o uso de dispositivo, sendo que o relato de dispositivos ( $9 \%$ da amostra) é exclusivo deste Aglomerado.

É nítida a diferença quanto à seleção dos dentes, sendo no Aglomerado 1 selecionados pelo técnico de prótese dentária e, no Aglomerado 2, essa seleção é feita pelo próprio CirurgiãoDentista. No número de sessões de provas clínicas, os dois Aglomerados se enquadram na realização de apenas uma sessão. E, por fim, quanto à remontagem dos modelos para ajuste oclusal, o Aglomerado 1 mostra a ausência da remontagem, enquanto que o Aglomerado 2 associou-se a um perfil heterogêneo.

\section{DISCUSSÃO}

Neste estudo, o levantamento de práticas adotadas pelos Cirurgiões-Dentistas para a confecção de próteses totais levou à quantificação dos métodos convencionais e simplificados que são usados, bem como delineou diferentes perfis dos profissionais avaliados. Conhecer o quanto os Dentistas de nosso país têm usado técnicas simplificadas pode gerar estratégias para sua formação continuada.

O gênero masculino foi predominante na amostra como um todo. Porém, o primeiro Aglomerado apresenta uma proporção menor de homens do que o segundo, associada a um menor tempo de graduação. Isso pode ter ocorrido em decorrência da feminilização da profissão com o passar dos anos, o que tem sido descrito por vários autores ${ }^{22-24}$. Conclusões de que Dentistas do gênero feminino adotam práticas específicas, contudo, devem 
ser vistas com cautela, já que as mulheres não consistiram em maioria da amostra, mesmo no primeiro Aglomerado.

A maior parte dos participantes tem como principal fonte de renda a prática clínica odontológica, sendo este um fator importante, pois garante a inclusão de Cirurgiões-Dentistas que exercem a clínica odontológica regularmente.

A apresentação de um perfil fechado sujeitando a amostra a profissionais restritos às clínicas privadas ou conveniadas limita a extrapolação dos resultados para Dentistas vinculados à saúde pública, uma vez que a sobrecarga da demanda espontânea, o trabalho repetitivo e o excesso de atendimentos clínicos são variáveis frequentemente apontadas por Cirurgiões-Dentistas que trabalham em âmbito público; nestas condições, práticas simplificadas poderiam ser preferidas ${ }^{25}$.

A literatura mostra maior satisfação profissional ligada ao maior tempo de trabalho, ou seja, maior tempo de formação, principalmente devido a questões como melhores rendimentos, maior reconhecimento profissional e melhor relação com o paciente ${ }^{26}$. Como este estudo obteve um perfil heterogêneo e o tempo de formação não diferiu importantemente nos dois Aglomerados, pode-se afirmar que essa variável não influenciou os resultados obtidos.

A análise inferencial demonstrou dois Aglomerados, sendo o primeiro (Aglomerado 1) composto por mais homens do que mulheres; o segundo (Aglomerado 2) também é composto por mais homens, no entanto com maior tempo de formação. No Aglomerado 1, foram prevalentes profissionais do gênero masculino e que parecem atender um maior número de pacientes ao dia. Isso sugere um atendimento em clínicas menos especializadas, se comparado ao Aglomerado 2. Como as horas semanais trabalhadas são parecidas nos dois Aglomerados, o segundo provavelmente é formado por profissionais menos comprometidos com grandes quantidades de atendimentos diários. Esse mesmo Aglomerado considera um número maior de sessões como sendo ideal para a confecção das próteses totais, pois possivelmente isto estaria mais próximo de suas condições de trabalho. Ainda, o Aglomerado 2 tem a clínica como principal fonte de renda, sugerindo um maior comprometimento com o atendimento odontológico, como citado acima.

O modo de seleção de dentes artificiais mostra claramente a discrepância entre os dois grupos: no Aglomerado 1, há uma tendência em delegar esta função ao Técnico de Prótese Dentária, enquanto que, no Aglomerado 2, é o próprio Cirurgião-Dentista quem faz essa seleção.

Nos dois grupos, é predominante a realização de duas moldagens para obtenção das próteses totais. Isso confronta relatos prévios quanto à utilização de apenas uma moldagem realizada por Dentistas da Inglaterra, adeptos ao procedimento simplificado no qual apenas um molde é obtido a partir de moldeiras de estoque, sem a necessidade na confecção de moldeiras individuais ${ }^{8}$.

A utilização do arco facial é bem distinta entre os dois grupos. No Aglomerado 2, a presença de profissionais mais especializados na área de Prótese Total sugere a valorização de aspectos técnicos em vez da praticidade no tratamento, sendo esse o grupo que relatou o registro utilizando o arco facial.

Ainda, o Aglomerado 2 lança mão de meios métricos para determinação da dimensão vertical de oclusão e faz a determinação do plano oclusal por meio de guias anatômicos, o que mais uma vez caracteriza este grupo pela utilização de técnicas convencionais para obtenção de próteses totais.

Quanto à remontagem dos modelos em articulador, os dois grupos tendem à utilização da técnica simplificada, ou seja, a não remontagem dos modelos. No entanto, quase todos os profissionais que responderam 'sim' para a remontagem estão incluídos no segundo grupo, tendenciando, mais uma vez, esse grupo à utilização de técnicas mais convencionais.

Os profissionais com menos especialidades tendem a realizar procedimentos mais resumidos e os especialistas, à utilização de técnicas convencionais, comportamento esse parecido ao dos graduandos de Odontologia, pois o procedimento convencional é o preconizado na confecção de próteses totais nas faculdades do Brasil e dos Estados Unidos ${ }^{6,7}$.

Dentre as limitações do estudo, pode-se citar o fato de a entrevista não ter sido feita pessoalmente, limitando o respondente a respostas fechadas e sem a possibilidade de esclarecer dúvidas, bem como a falta no controle da resposta do indivíduo que responderia à pesquisa. Em contrapartida, esse método de obtenção de respostas on line abrangeu uma maior quantidade de respondentes, a heterogeneidade dos mesmos e o alcance a profissionais residentes em diferentes regiões do país.

Ainda que a maioria dos Cirurgiões-Dentistas entrevistados realize técnicas por vezes simplificadas e, na maioria das vezes, sem base científica fundamentada, ou seja, apenas pela própria experiência clínica, muitos são os relatos na literatura que descrevem o método simplificado como capaz de garantir próteses totais satisfatórias em todos os aspectos ${ }^{12-18,27}$.

\section{CONCLUSÃO}

Os profissionais selecionados no estudo apresentaram dois perfis, sendo um formado por generalistas adeptos de perfis mais simplificados e outro formado por profissionais adeptos de técnicas convencionais.

\section{AUXÍLIOS FINANCEIROS}

Os autores agradecem à FAPESP pelo apoio financeiro (Bolsa IC, Processo n. ${ }^{\circ}$ 11/09364-0). 


\section{REFERÊNCIAS}

1. Felton DA. Edentulism and comorbid factors. J Prosthodont. 2009 Feb;18(2):88-96. http://dx.doi.org/10.1111/j.1532-849X.2009.00437.x

2. Petersen PE, Bourgeois D, Ogawa H, Estupinan-Day S, Ndiaye C. The global burden of oral diseases and risks to oral health. Bull World Health Organ. 2005 Sep;83(9):661-9.

3. Brasil. Ministério da Saúde. Projeto SB Brasil 2010: resultados principais. Brasília: Ministério da Saúde; 2011.

4. Rahn AO, Heartwell Jr CM. Textbook of complete dentures. $5^{\text {th }}$ ed. Philadelphia: Lea and Febiger; 1993.

5. Zarb GA, Bolender CL, Eckert S, Jacob R, Fenton A, Jacob R. Prosthodontic treatment for edentulous patients: complete dentures and implant-supported prostheses. $12^{\text {th }}$ ed. St. Louis: Elsevier; 2003.

6. Souza RF, Leles CR, Compagnoni MA. A survey of complete denture teaching in Brazilian dental schools. Pos-Grad Rev Fac Odontol São José dos Campos. 2002 Jan-Apr;5(1):5-11.

7. Rashedi B, Petropoulos VC. Preclinical complete dentures curriculum survey. J Prosthodont. 2003 Mar;12(1):37-46.

8. Duncan JP, Taylor TD. Teaching an abbreviated impression technique for complete dentures in an undergraduate dental curriculum. J Prosthet Dent. 2001 Feb;85(2):121-5. http://dx.doi.org/10.1067/mpr.2001.113699

9. Nascimento DFF, Patto RBL, Marchini L, Cunha VPP. Double-blind study for evaluation of complete dentures made by two techniques with and without facebow. Braz J Oral Sci. 2004 Apr/Jun;3(9):439-45.

10. Sutton AF, Glenny AM, McCord JF. Interventions for replacing missing teeth: denture chewing surface designs in edentulous people. Cochrane Database Syst Rev. 2005 Jan 25;(1):CD004941.

11. Ellis JS, Pelekis ND, Thomason JM. Conventional rehabilitation of edentulous patients: the impact on oral health-related quality of life and patient satisfaction. J Prosthodont. 2007 Jan-Feb;16(1):37-42. http://dx.doi.org/10.1111/j.1532-849X.2006.00152.x

12. Della Vecchia MP, Regis RR, Cunha TR, de Andrade IM, da Matta JC, de Souza RF. A randomized trial on simplified and conventional methods for complete denture fabrication: cost analysis. J Prosthodont. 2013 Jul 26. [Epub ahead of print]. http://dx.doi.org/10.1111/ jopr.12090

13. Cunha TR, Della Vecchia MP, Regis RR, Ribeiro AB, Muglia VA, Mestriner W Jr, et al. A randomised trial on simplified and conventional methods for complete denture fabrication: masticatory performance and ability. J Dent. 2013 Feb;41(2):133-42. http://dx.doi.org/10.1016/j. jdent.2012.09.008

14. Heydecke G, Vogeler M, Wolkewitz M, Turp JC, Strub JR. Simplified versus comprehensive fabrication of complete dentures: patient ratings of denture satisfaction from a randomized crossover trial. Quintessence Int. 2008 Feb;39(2):107-16.

15. Kawai Y, Murakami H, Shariati B, Klemetti E, Blomfield JV, Billette L, et al. Do traditional techniques produce better conventional complete dentures than simplified techniques? J Dent. 2005 Sep;33(8):659-68. http://dx.doi.org/10.1016/j.jdent.2005.01.005

16. Kawai Y, Murakami H, Takanashi Y, Lund JP, Feine JS. Efficient resource use in simplified complete denture fabrication. J Prosthodont. 2010 Oct;19(7):512-6. http://dx.doi.org/10.1111/j.1532-849X.2010.00628.x

17. Omar R, Al-Tarakemah Y, Akbar J, Al-Awadhi S, Behbehani Y, Lamontagne P. Influence of procedural variations during the laboratory phase of complete denture fabrication on patient satisfaction and denture quality. J Dent. 2013 Aug 1. doi:pii: S0300-5712(13)00186-3. [Epub ahead of print].

18. Regis RR, Cunha TR, Della Vecchia MP, Ribeiro AB, Silva-Lovato CH, de Souza RF. A randomised trial of a simplified method for complete denture fabrication: patient perception and quality. J Oral Rehabil. 2013 Jul;40(7):535-45. http://dx.doi.org/10.1111/joor.12063

19. Clark DM, Oyen OJ, Feil P. The use of specific dental school-taught restorative techniques by practicing clinicians. J Dent Educ. 2001 Aug;65(8):760-5.

20. Hyde TP, McCord JF. Survey of prosthodontic impression procedures for complete dentures in general dental practice in the United Kingdom. J Prosthet Dent. 1999 Mar;81(3):295-9. http://dx.doi.org/10.1016/S0022-3913(99)70272-6

21. Nunes MF, Silva ET, Santos LB, Queiroz MG, Leles CR. Profiling alumni of a Brazilian public dental school. Hum Resour Health. 2010 Aug; 18;8:20.

22. Pereira MF, Botelho TL. Perfil do cirurgião-dentista no estado de Goiás. Rev Fac Odontol UFG. 1997;1(1):37-40.

23. Silva SRC, Rosa AGF. Características dos cirurgiões dentistas com vínculo empregatício em empresas públicas e/ou privadas no município de Araraquara-SP. Rev Odontol UNESP. 1996 jan-dez;25(n.esp):9-18.

24. Mott ML, Alves OSF, Muniz MA, Martino LVS, Santos APF, Maestrini K. 'Moças e senhoras dentistas': formação, titulação e mercado de trabalho nas primeiras décadas da República. Hist Ciênc Saúde-Manguinhos. 2008 jun.;15(suppl.):97-116. http://dx.doi.org/10.1590/ S0104-59702008000500005

25. Chaves MC, Miranda, AS. Discursos de cirurgiões-dentistas do Programa Saúde da Família: crise e mudança de habitus na Saúde Pública. Interface (Botucatu). 2008 jan-mar;12(24):153-67. http://dx.doi.org/10.1590/S1414-32832008000100012

26. Shugars DA, DiMatteo MR, Hays RD, Cretin S, Johnson JD. Professional satisfaction among California general dentists. J Dent Educ. 1990 Nov;54(11):661-9.

27. Nuñez MCO, Silva DC, Barcelos BA, Leles CR. Patient satisfaction and oral health-related quality of life after treatment with traditional and simplified protocols for complete denture construction. Gerodontology 2013 Oct 21. [Epub ahead of print]. 
Os autores declaram não haver conflitos de interesse.

\section{AUTOR PARA CORRESPONDÊNCIA}

Raphael Freitas de Souza

Departamento de Materiais Dentários e Prótese, Faculdade de Odontologia de Ribeirão Preto, USP - Universidade de São Paulo, Av. do Café, s/n, Bairro Monte Alegre, 14040-904 Ribeirão Preto - SP, Brasil

e-mail: raphael@forp.usp.br

Recebido: Outubro 16, 2013

Aprovado: Dezembro 12, 2013 